Pacific Journal of Mathematics

LAss OF WaLLMAN-TYP Extension 


\title{
A CLASS OF WALLMAN-TYPE EXTENSIONS
}

\author{
ELLen E. REed
}

This paper grew out of an attempt to determine what $T_{2}$-compactifications, the Wallman compactification, and the one-point compactification have in common. It turns out that in each case the associated nearness is generated by those grills which contain ultraclosed filters and are clans with respect to the associated proximity. Such a nearness will be called a Wallman nearness, and this paper is a study of the properties of Wallman nearnesses and their extensions.

A mild "covering" condition on a proximity guarantees that it contains a Wallman nearness. Each covered proximity contains exactly one Wallman nearness. This sets up a 1-1 correspondence between covered proximities and extensions obtained from Wallman nearnesses. The latter will be called Wallman-type extensions. These can be characterized by the fact that they are covered extensions and satisfy a certain completeness property; namely, the duals of certain clans must converge. This summarizes the first two sections.

The last section is a study of compact Wallman-type extensions. A condition on the proximity is obtained which guarantees that the associated Wallman-type extension is compact. The condition states that certain very large grills containing ultraclosed filters must be clans with respect to the given proximity. Such a proximity will be called a compactification proximity. It turns out that compactification proximities give rise to weakly regular compactifications. The paper ends with a study of the relation between weak regularity and Wallman-type extensions.

O. Preliminaries. The paper uses the definitions and notation of Reed [3] and Thron [5]. Throughout the paper we will assume that $X$ is a fixed $T_{1}$-space and that $\pi$ is a Lodato proximity compatible with the topology on $X$.

1. Wallman-type nearnesses. Out of the large class of nearnesses compatible with a given Lodato proximity $\pi$ we will choose one of particular interest. It is generated by those $\pi$-clans which contain ultraclosed filters. We will call this the Wallman nearness generated by $\pi$. A mild condition on $\pi$ guarantees that the Wallman nearness it generates is actually in the same proximity class. Such proximities will be called covered proximities.

In this section we will develop some properties of Wallman 
nearnesses, and in fact give a characterization of these nearnesses. In particular we will see that each covered proximity has exactly one Wallman nearness in its proximity class.

Definition 1.1. A Wallman $\pi$-clan is a $\pi$-clan which contains some ultraclosed filter.

Proposition 1.2. The set of all Wallman $\pi$-clans generates a nearness on $X$. This nearness consists of all families of subsets of $X$ which are subsets of some Wallman $\pi$-clan.

Proof. By a theorem of Gagrat and Thron [1, Thm. 2.7], all we need to establish is that each singleton $\{x\}$ is in some Wallman $\pi$-clan. In fact it is easy to see that each point filter $\dot{x}$ is itself a Wallman $\pi$-clan. Clearly $\dot{x}$ is at least a $\pi$-clan. Since $X$ is $T_{1}$, we have that $\dot{x}$ is an ultraclosed filter.

NotATION AND DEFINITION 1.3. Let the nearness generated by the Wallman $\pi$-clans be denoted by $\nu_{W}(\pi)$, or by $\nu_{W}$ when the meaning is clear. By a Wallman nearness on $X$ we will mean any nearness of the form $\nu_{W}(\pi)$, where $\pi$ is a Lodato proximity compatible with $X$.

Next we will establish that the usual construction of an extension from a nearness is a 1-1 map on the Wallman nearnesses compatible with $X$.

THEOREM 1.4. If $\nu$ is a Wallman nearness on $X$ then $\nu$ is a cluster-generated Lodato nearness compatible with the topology on $X$.

Proof. Assume $\nu=\nu_{W}(\pi)$, where $\pi$ is a Lodato proximity compatible with the topology $\mathscr{T}$ on $X$. To establish that $\nu$ is compatible with $\mathscr{T}$, note that for $x \in X$ the grill $\sigma_{x}=\left\{A \subset X \mid x \in A^{-}\right\}$ is a Wallman $\pi$-clan. To see that $\nu$ is a Lodato nearness, use the fact that if $\sigma$ is a $\pi$-clan then $b \sigma=\left\{A \subset X \mid A^{-} \in \sigma\right\}$ is a $\pi$-clan. Finally, to obtain the result that $\nu$ is cluster-generated, observe that every $\pi$-clan is contained in a maximal $\pi$-clan. But every maximal $\pi$-clan in $\nu$ is a $\nu$-cluster.

REMARK 1.5. In Reed [3] a construction was described whereby a Lodato nearness $\nu$ compatible with the topology on a $T_{1}$-space gave rise to a principal $T_{1}$-extension $\kappa_{\nu}$. Further it was established that this map $\nu$ to $\kappa_{\nu}$ was $1-1$ on cluster generated Lodato nearnesses.

Thus the preceding result allows us to conclude that the 
Wallman nearnesses on $X$ map in a 1-1 fashion into the principal $T_{1}$-extensions of $X$.

In what follows we will show that a proximity class contains at most one Wallman nearness. We will also obtain a necessary and sufficient condition for a proximity class to contain a Wallman nearness.

Proposition 1.6. If $\nu$ is a Wallman nearness on $X$ then $\pi_{\nu}$ is a Lodato proximity compatible with the topology on $X$, and $\nu=\nu_{W}\left(\pi_{\nu}\right)$.

Proof. By Theorem 1.4 if $\nu$ is a Wallman nearness on $X$ then $\nu$ is Lodato and compatible with $\mathscr{T}$. Hence the same holds true for $\pi_{\nu}$. Now suppose $\nu=\nu_{W}(\pi)$. We wish to show $\nu_{W}(\pi)=\nu_{W}\left(\pi_{\nu}\right)$. This holds, provided every Wallman $\pi$-clan is a Wallman $\pi_{\nu}$-clan, and conversely.

(1) $\pi_{\nu} \subset \pi$. If $A \pi_{\nu} B$ then $A$ and $B$ are members of the same $\pi$-clan $\sigma$. This implies $A \pi B$.

(2) Every $\pi_{\nu}$-clan is a $\pi$-clan. This follows immediately from (1).

(3) Every Wallman $\pi$-clan is a $\pi_{\nu}$-clan. If $\sigma$ is a Wallman $\pi$-clan then by definition $\sigma \in \nu_{W}(\pi)$, which is $\nu$. Thus $\sigma$ is a $\pi_{\nu}$-clan.

THEOREM 1.7. A proximity class contains at most one Wallman nearness.

Proof. This follows easily from Proposition 1.6.

Next we will obtain a condition on $\pi$ which guarantees that $\nu_{W}(\pi)$ is in the proximity class of $\pi$.

Definition 1.8. A proximity $\pi$ is covered iff any two $\pi$-near sets are members of the same Wallman $\pi$-clan.

THEOREM 1.9. The following conditions are equivalent.

(1) The Lodato proximity $\pi$ is covered.

(2) The nearness $\nu_{W}(\pi)$ is compatible with $\pi$.

(3) The class of $\pi$ contains a Wallman nearness.

Proof. $\quad(1) \Rightarrow(2)$. Let $\nu_{W}(\pi)$ be denoted by $\nu$. From the definition of $\nu$ it is clear that $\pi_{\nu} \subset \pi$. If $\pi$ is covered, we obtain $\pi \subset \pi_{\pi}$.

$(2) \Longrightarrow(3)$. This follows from the fact that $\nu_{W}(\pi)$ is a Wallman nearness.

$(3) \Rightarrow(1)$. Let $\nu$ be a Wallman nearness in the class of $\pi$. Then by Prop. 1.6 we have $\nu=\nu_{w}(\pi)$. From this it is easy to see that $\pi$ is covered. 
REMARK 1.10. Every Lodato proximity compatible with $\mathscr{T}$ is near a covered proximity. For if $\pi$ is not covered and $\nu=\nu_{W}(\pi)$ then $\pi_{\nu}$ is covered, since $\nu$ is a Wallman nearness in its proximity class. But $\pi$ is close to $\pi_{\nu}$, since both proximities have the same Wallman $\pi$-clans. (See the proof of Prop. 1.6.) In fact it may be that all Lodato proximities are covered. I was unable to find a counterexample.

In any case we lose nothing by considering only covered proximities, since every Wallman nearness is in the class of some covered proximity.

In what follows we will obtain a characterization of Wallman nearnesses.

DeFinITION 1.11. A nearness $\nu$ compatible with $\mathscr{T}$ is covered iff for $\mathscr{A} \in \nu$ there is an ultraclosed filter $\mathscr{C}$ such that $\mathscr{\mathscr { C }} \cup \mathscr{\mathscr { C }}$ is in $\nu$.

THEOREM 1.12. Let $\nu$ be a Lodato nearness compatible with $\mathscr{T}$. Then $\nu$ is Wallman iff $\nu$ is covered and contains every Wallman $\pi_{\nu}$-clan.

Proof. Let $\nu$ be a Lodato nearness compatible with $\mathscr{T}$. If $\nu$ is Wallman then by Proposition 1.6 we have $\nu=\nu_{W}\left(\pi_{\nu}\right)$. Clearly then $\nu$ is covered and contains every Wallman $\pi_{\nu}$-clan.

Conversely, suppose $\nu$ is covered and contains every Wallman $\pi_{\nu}$-clan. We will show that $\nu=\nu_{W}\left(\pi_{\nu}\right)$. Note $\pi_{\nu}$ is Lodato and compatible with $\mathscr{T}$, since the same holds for $\nu$. Now $\nu$ contains all Wallman $\pi_{\nu}$-clans and so we have $\nu_{W}\left(\pi_{\nu}\right) \cong \nu$.

Containment the other way follows from the fact that $\nu$ is covered and that every proximity class $\pi$ has a largest member $\nu_{G}(\pi)$ which is generated by all the $\pi$-clans. (See Reed [3], Thm. 2.7.) If $\mathscr{A} \in \nu$ then there is an ultraclosed filter $\mathscr{C}$ such that $\mathscr{A} \cup$ $\mathscr{\mathscr { C }} \in \nu$. Then $\mathscr{A} \cup \mathscr{C} \in \nu_{G}\left(\pi_{\nu}\right)$, and so there is a $\pi_{\nu}$-clan $\sigma$ such that $\mathscr{A} \cup \mathscr{C} \in \sigma$. Clearly $\sigma$ is then a Wallman $\pi_{\nu}$-clan containing $\mathscr{A}$, so that $\mathscr{A} \in \mathcal{\nu}_{W}\left(\pi_{\nu}\right)$.

2. Wallman-type extensions. In this section we will investigate the properties of Wallman-type extensions, which are extensions obtained from Wallman nearnesses. These are characterized by the property that they are covered and satisfy a certain completeness property, which we will call Wallman-completeness. The determining factor for the Wallman-completeness of a principal 
$T_{1}$-extension is whether or not the induced nearness contains all Wallman $\pi$-clans.

It turns out that Wallman-type extensions are in 1-1 correspondence with covered Lodato proximities. Thus in constructing a Wallman-type extension we are choosing a particular nearness in the proximity class and obtaining the standard extension for that nearness. Many of the usual compactifications are obtained in this way from appropriately chosen proximities. These include the usual Wallman compactification, the Alexandroff one-point compactification, and all $T_{2}$-compactifications.

Definition 2.1. An extension $\kappa$ of $X$ is a Wallman-type extension iff there is a Wallman nearness $\nu$ on $X$ such that $\kappa$ is equivalent to $\operatorname{Ext}(\nu)$, the extension obtained in the usual way from $\nu$. (See Reed [3], Construction 1.15.)

Definition 2.2. Let $\kappa=(e, Y)$ be an extension of $X$. A clan on $\kappa$ is a grill on $Y$ which is a clan with respect to the usual proximity on $\kappa$; namely, two sets are near if their closures intersect. A Wallman clan on $\kappa$ is a clan which contains the image under $e$ of an ultraclosed filter on $X$. Finally, we say $\kappa$ is a Wallman-complete iff the dual of every Wallman clan converges.

The next two results are technical results which will be useful later.

Proposition 2.3. If $\kappa=\operatorname{Ext}(\nu)$ where $\nu$ is a Wallman nearness on $X$, then $\nu=\nu_{\kappa}$ and $\pi_{\nu}=\pi_{\kappa}$.

Proof. By Theorem 1.4 we have that $\nu$ is cluster-generated, Lodato, and compatible with $\mathscr{T}$. Therefore the nearness induced by $\kappa$ is the original nearness $\nu$. (See Reed [3], Thm. 1.18.) Thus $\nu=\nu_{\kappa}$, and from this it follows that $\pi_{\nu}=\pi_{\kappa}$.

Corollary 2.4. If $\kappa$ is a Wallman-type extension then $\nu_{\kappa}=$ $\nu_{W}\left(\pi_{\kappa}\right)$.

Proof. The proof follows readily from Proposition 2.3, Proposition 1.6, and the fact that equivalent extensions of $X$ induce the same nearness on $X$, and hence the same proximity on $X$.

Next we will show that a principal extension is Wallman-complete iff the induced nearness is large enough to include the associated Wallman nearness. 
THEOREM 2.5. Let $\kappa=(e, Y)$ be a principal extension of $X$. Then $\kappa$ is Wallman-complete iff $\nu_{W}\left(\pi_{\kappa}\right) \subset \nu_{\kappa}$.

Proof. $(\Rightarrow)$ Suppose $\kappa$ is Wallman-complete. Let $\sigma$ be a Wallman $\pi_{\kappa}$-clan on $X$. We will show that for some $y \in Y$ we have $\sigma \subset \tau(y)$.

Since $\sigma$ is a Wallman $\pi_{k}$-clan it is easy to check that $e \sigma$ is a Wallman clan on $Y$. But $\kappa$ is Wallman-complete, and so the dual of $e \sigma$ converges to some $y$ in $Y$. It is easy to check that $\sigma \subset \tau(y)$.

$\Leftrightarrow$ Suppose $\nu_{w}\left(\pi_{k}\right) \subset \nu_{\kappa}$. Let $\mu$ be a Wallman clan on $Y$. We wish to show $d \mu$ converges.

Let $\sigma=\left\{A: e(A)^{-} \in \mu\right\}$. Then it can easily be verified that $\sigma$ is a Wallman $\pi_{\kappa}$-clan. By our assumption on $\nu_{\kappa}$ we have $\sigma \in \nu_{\kappa}$. Choose $z \in Y$ so $\sigma \subset \tau(z)$. Then since $\kappa$ is a principal extension of $X$ we have that $d \mu \rightarrow z$.

COROLlARY 2.6. Wallman-type extensions are Wallman-complete.

Proof. Let $\kappa$ be a Wallman-type extension. Then $\nu_{\kappa}=\nu_{W}\left(\pi_{\kappa}\right)$, by Corollary 2.4. From the preceding theorem it now follows that $\kappa$ is Wallman-complete.

It turns out that Wallman-type extensions are characterized by being Wallman-complete and covered.

DEFINITION 2.7. An extension of $\kappa$ of $X$ is covered iff every point of $\kappa$ is the limit of the image of an ultraclosed filter in $X$.

Proposition 2.8. Wallman-type extensions are covered.

Proof. Let $\kappa$ be equivalent to $\lambda=\operatorname{Ext}(\nu)$, where $\nu$ is a Wallman nearness on $X$. It is easy to see that $\kappa$ is covered iff $\lambda$ is covered. To show $\lambda$ is covered let $\sigma$ be any $\nu$-cluster. We need to show there is an ultraclosed filter $\mathscr{U}$ such that $e_{\nu}(\mathscr{C}) \rightarrow \sigma$.

Since $\nu$ is a Wallman nearness, it is Lodato and it is compatible with $\mathscr{T}$ (Thm. 1.4). Therefore Theorem 1.12 applies, and we can say $\nu$ is covered. Since $\sigma \in \nu$ there is an ultraclosed filter $\mathscr{C}$ such that $\sigma \cup \mathscr{C} \in \nu$. But $\sigma$ is a $\nu$-cluster, so $\mathscr{\mathscr { C }} \subset \sigma$. We claim that $e_{\nu}(\mathscr{U}) \rightarrow \sigma$.

Recall the closed sets of $\lambda$ have as a base all sets of the form $A^{\nu}$, where $A$ is a closed set in $X$. By $A^{\nu}$ is meant the set of all $\nu$-clusters containing $A$. Suppose $A$ is closed in $X$ and $\sigma \notin A^{\nu}$. We need to show $\sim A^{\nu}$ is in $e_{\nu}(\mathscr{Q})$.

Since $\sigma \notin A^{\nu}$ we have $A \notin \sigma$ and so $A \notin \mathscr{C}$. But $\mathscr{W}$ is ultraclosed, so there is a closed set $F$ in $\mathscr{C}$ such that $F \cap A=\varnothing$. We claim that $e_{\nu}(F) \subset \sim A^{\nu}$. 
Let $x \in F$. Recall $e_{\nu}(x)=\left\{B \mid x \in B^{-}\right\}$. Since $x \in F$ we have $x \notin A$ and so $A \notin e_{\nu}(x)$. (Recall $A$ is closed.) Thus $e_{\nu}(x) \in \sim A^{\nu}$ as desired.

THEOREM 2.9. Let $\kappa$ be a principal $T_{1}$-extension of $X$. The following conditions are equivalent:

(1) $\kappa$ is a Wallman-type extension.

(2) $\kappa$ is covered and Wallman-complete.

(3) $\nu_{\kappa}=\nu_{w}\left(\pi_{\kappa}\right)$.

Proof. $\quad(3) \Rightarrow(1)$. Let $\nu=\nu_{W}\left(\pi_{\kappa}\right)$. We wish to show $\nu$ is a Wallman nearness on $X$ and $\kappa$ is equivalent to Ext ( $\nu$ ).

It is easy to check that $\pi_{\kappa}$ is a compatible Lodato proximity on $X$, so that $\nu$ is indeed a Wallman nearness on $X$.

Now since $\kappa$ is a principal $T_{1}$-extension of $X$ we have that $\kappa$ is equivalent to $\operatorname{Ext}\left(\nu_{\kappa}\right)$. (See Reed [3], Thm. 1.19.) But $\nu=\nu_{\kappa}$, by assumption, and so we have the desired equivalence.

$(1) \Rightarrow(2)$. This follows immediately from Corollary 2.6 and Proposition 2.8 .

$(2) \Rightarrow(3)$. Let $\kappa=(e, Y)$. First we will show that each $\tau(y)$ is a Wallman $\pi_{\kappa}$-clan. Clearly $\tau(y)$ is at least a $\pi_{\kappa}$-clan. Since $\kappa$ is covered we can choose an ultraclosed filter $\mathscr{C}$ such that $e(\mathscr{C}) \rightarrow y$. It is easy to check that $\mathscr{U} \subset \tau(y)$, so that $\tau(y)$ is Wallman. Thus $\nu_{\kappa} \subset \nu_{W}\left(\pi_{\kappa}\right)$.

Now let $\sigma$ be a Wallman $\pi_{\kappa}$-clan. Then $e \sigma$ is a Wallman clan on $Y$. Since $\kappa$ is Wallman-complete, the dual of $e \sigma$ converges to some $y$ in $Y$. It is straightforward to check that $\sigma \subset \tau(y)$.

Notation AND REMARK 2.10. For $\pi$ a Lodato proximity on $X$ let $\kappa_{W}(\pi)$ denote $\operatorname{Ext}\left(\nu_{W}(\pi)\right)$, the associated Wallman-type extension. We can regard $\kappa_{W}$ as a map from proximities to extensions, and regard $\pi$ as a map from extensions to their induced proximities. (So $\pi(\kappa)=\pi_{\kappa}$ the induced proximity.) If we limit ourselves to covered Lodato proximities and Wallman-type extensions then $\kappa_{W}$ and $\pi$ are inverses of each other. This is the content of the next theorem.

THEOREM 2.11.

(1) Let $\pi$ be a covered Lodato proximity on $X$ and let $\kappa=\kappa_{W}(\pi)$. Then $\pi_{\kappa}=\pi$.

(2) Let $\kappa$ be a Wallman-type extension of $X$. Then $\kappa_{W}\left(\pi_{\kappa}\right)$ is equivalent to $\kappa$.

Proof. (1) Let $\pi$ be a covered Lodato proximity on $X$. Let $\nu=\nu_{W}(\pi)$ and let $\kappa=\kappa_{W}(\pi)=\operatorname{Ext}(\nu)$. Since $\pi$ is covered, we have 
that $\pi=\pi_{\nu}$. Also $\nu=\nu_{\kappa}$, by Theorem 1.5. Thus we have $\pi=\pi_{\nu}=$ $\pi_{\nu_{\kappa}}=\pi_{\kappa}$.

(2) Let $\kappa$ be a Wallman-type extension of $X$. From the preceding theorem we have $\nu_{\kappa}=\nu_{W}\left(\pi_{\kappa}\right)$. Thus $\operatorname{Ext}\left(\nu_{\kappa}\right)=\kappa_{W}\left(\pi_{\kappa}\right)$. But $\operatorname{Ext}\left(\nu_{\kappa}\right)$ is equivalent to $\kappa$, since $\kappa$ is a principal $T_{1}$-extension of $X$. (See Reed [3], Thm. 1.19).

Lemma 2.12. Let $\kappa=(e, Y)$ be a covered extension of $X$. Then $\pi_{\kappa}$ is covered, and in fact each $\tau(y)$ is a Wallman $\pi_{\kappa}$-clan.

Proof. Recall $A \pi_{x} B$ iff there is a $y \in Y$ such that $A, B \in \tau(y)$. Thus to show $\pi_{\kappa}$ is covered it is sufficient to show that each $\tau(y)$ is a Wallman $\pi_{\kappa}$-clan. Clearly $\tau(y)$ is a $\pi_{\kappa}$-clan. Since $\kappa$ is covered, we can choose an ultraclosed filter $\mathscr{C}$ on $X$ such that $e(\mathscr{C}) \rightarrow y$. It is easy to check that $\mathscr{U} \subset \tau(y)$, so that $\tau(y)$ is a Wallman clan.

COROLLARY 2.13. The map $\kappa_{W}$ is a bijection from the covered Lodato proximities on $X$ to the covered Wallman-complete principal $T_{1}$-extensions of $X$.

Proof. Let $\pi$ be any covered Lodato proximity on $X$. Clearly $\kappa_{W}(\pi)$ is a Wallman-type extension, so by Theorem 2.9 we have that $\kappa_{W}(\pi)$ is covered and Wallman-complete.

Now suppose $\kappa$ is any covered Wallman-complete principal $T_{1^{-}}$ extension of $X$. By Theorem 2.9, $\kappa$ is a Wallman-type extension. Hence by the preceding theorem, $\kappa$ is equivalent to $\kappa_{W}\left(\pi_{\kappa}\right)$. Since $\kappa$ is covered, $\pi_{\kappa}$ is covered, by the preceding lemma. Thus $\kappa$ can be considered in the image of $\kappa_{W}$.

Finally, let $\pi_{1}$ and $\pi_{2}$ be covered Lodato proximities on $X$, and suppose $\kappa_{W}\left(\pi_{1}\right)$ is equivalent to $\kappa_{W}\left(\pi_{2}\right)$. Then the induced proximities are $\pi_{1}$ and $\pi_{2}$ respectively, by Theorem 2.11. But equivalent extensions induce the same nearness, and hence the same proximity. (See Reed [3], Lemma 1.9.) Thus $\pi_{1}=\pi_{2}$.

REMARK 2.14. It has been shown that the principal $T_{1}$-extensions of $X$ are in 1-1 correspondence with certain nearnesses on $X$. (See Reed [3], Cor. 1.20.) In general a proximity class contains many nearnesses. Thus to obtain a 1-1 map from proximities to extensions it is necessary to pick out a particular nearness. What we have done here is to obtain a way to pick a nearness out of a proximity class; namely, by choosing the $\pi$-clans which contain ultraclosed filters. This particular choice is of interest because it includes so many of the known compactifications. This will be seen 
in the next theorem.

THEOREM 2.15. The following compactifications are Wallmantype extensions:

(1) all $T_{2}$-compactifications;

(2) the usual Wallman compactification of a $T_{1}$-space;

(3) the one-point compactification of a $T_{1}$-space.

Proof. First we will establish that all these compactifications are Wallman-complete. Let $\kappa=(e, Y)$ be a $T_{1}$-compactification of $X$. Let $\sigma$ be a Wallman clan in $\kappa$. Pick $\mathscr{W}$ an ultraclosed filter on $X$ such that $e \mathscr{U} \subset \sigma$. Since $Y$ is compact, $e(\mathscr{U}) \rightarrow y$ for some $y$ in $Y$. We claim that the dual $d \sigma$ converges to $y$, provided $\kappa$ is in one of the above categories. Let $V$ be an open neighborhood of $y$. The idea of the proof is to find a set $S$ in $\sigma$ which is far from $\sim V$. Clearly in that case $V \in d \sigma$.

(1) Suppose $Y$ is Hausdorff. Then it is regular, and we can choose $W$ open such that $y \in W$ and $W^{-} \subset V$. Then $W$ is far from $\sim V$. Since $e \mathscr{U} \rightarrow y$ we have $W \in e \mathscr{U} \subset \sigma$.

(2) Suppose $\kappa$ is the usual Wallman compactification of $X$. Since $\kappa$ is a principal extension, we can choose a closed subset $A$ of $X$ such that $y \in \sim e(A)^{-} \subset V$. Since $y \notin e(A)^{-}$and $e(\mathscr{C}) \rightarrow y$ we have that $A \notin \mathscr{W}$. But $\mathscr{W}$ is ultraclosed, so $\sim A \in \mathscr{Q}$. Let $K$ be a closed set in $\mathscr{C}$ such that $K \subset \sim A$. We claim that $e K$ is far from $\sim V$. Note $e(K) \in e \mathscr{Q} \subset \sigma$.

Now since $\kappa$ is the usual Wallman compactification, and $K \cap A=$ $\varnothing$, we have $e(K)^{-} \cap e(A)^{-}=\varnothing$. Thus $e(K)^{-} \subset V$. This confirms that $e(K)$ is far from $\sim V$.

(3) Now suppose $\kappa$ is the usual 1-point compactification of $X$. If $\mathscr{C}$ is a convergent filter then since $\mathscr{C}$ is ultraclosed we have $\mathscr{C}=\dot{x}$ for some $x \in X$. Since $e \mathscr{C} \rightarrow y$ we have $y=e(x)$. Here we used that $Y$ is a $T_{1}$-space. Thus $\{y\}$ is a set in $\sigma$ which is far from $\sim V$.

Suppose now that $\mathscr{U}$ does not converge. Since $e(\mathscr{C}) \rightarrow y$ we have that $y \notin e(X)$. For if $e(\mathscr{C}) \rightarrow e(x)$ then $\mathscr{C} \rightarrow x$. Thus $y=\omega$ the "point at infinity". Now since $V \in e \mathscr{U}$ we can choose a closed set $K$ in $\mathscr{C}$ such that $K \subset e^{-1}(V)$. Then $e(K)^{-} \subset e(K) \cup\{\omega\} \subset V$. Thus $e(K)$ is far from $\sim V$. Note $e(K) \in e(\mathscr{C}) \subset \sigma$.

Now we need to establish that each of the listed compactifications is covered.

(1) Suppose $Y$ is a $T_{2}$-space. Let $y \in Y$, and consider $e^{-1}\left(\mathscr{N}_{y}\right)$. Since $Y$ is a compact $T_{2}$-space, it is regular. Thus $e^{-1}\left(\mathscr{N}_{y}\right)$ is a closed filter on $X$. By Zorn's lemma it must be contained in some ultraclosed filter $\mathscr{U}$. It is easy to check that $e(\mathscr{C}) \rightarrow y$. 
(2) Suppose $\kappa$ is the usual Wallman compactification of $X$. Then we can think of a point in $Y$ as an ultraclosed filter $\mathscr{U}$. By construction, $e(\mathscr{U}) \rightarrow \mathscr{U}$.

(3) Finally, suppose $\kappa$ is the 1-point compactification of $X$. Note that $X$ and $Y$ are $T_{1}$-spaces but they need not be $T_{2}$-spaces. Let $y \in Y$. If $y=e(x)$ then $\dot{x}$ is an ultraclosed filter for which $e(\dot{x}) \rightarrow y$. Suppose $y=\omega$, the point at infinity. Then $X$ is not compact and so there is a nonconvergent ultraclosed filter $\mathscr{C}$ on $X$. We claim that $e(\mathscr{U}) \rightarrow \omega$.

Let $G$ be an open neighborhood of $\omega$. Then by construction $\sim e^{-1}(G)$ is closed and compact. Since $\mathscr{C}$ is nonconvergent, $\sim e^{-1}(G)$ cannot be in $\mathscr{U}$. But $\mathscr{U}$ is maximal closed, so $e^{-1}(G) \in \mathscr{U}$. Thus $G \in e(\mathscr{U})$ as desired.

REMARK 2.16. From what we have just seen, many compactifications can be thought of as special cases of Wallman-type extensions. The kind of extension obtained depends on the proximity chosen. For example, if the proximity is an Efremovich proximity then the corresponding extension is a $T_{2}$-compactification. This is spelled out in the next theorem.

Notation 2.17. (1) Let $\pi_{W}$ denote the "Wallman" proximity on $X$ :

$$
A \pi_{W} B \text { iff } A^{-} \cap B^{-} \neq \varnothing
$$

(2) Let $\pi_{A}$ denote the "Alexandroff" proximity:

$A \pi_{A} B$ iff $A^{-} \cap B^{-} \neq \varnothing$ or else both $A^{-}$and $B^{-}$are noncompact sets.

THEOREM 2.18.

(1) If $\pi$ is an Efremovich proximity then $\kappa_{W}(\pi)$ is a $T_{2}$-compactification of $X$.

(2) The extension $\kappa_{W}\left(\pi_{W}\right)$ is the usual Wallman compactification of $X$. $X$.

(3) The extension $\kappa_{W}\left(\pi_{A}\right)$ is the one-point compactification of

Proof. Suppose $\kappa$ is either a $T_{2}$-compactification of $X$, or the usual Wallman compactification, or the one-point compactification of $X$. Then by Theorem 2.15 we have that $\kappa$ is a Wallman-type extension of $X$. Thus $\kappa$ is equivalent to $\kappa_{W}\left(\pi_{\kappa}\right)$, by Theorem 2.11. The idea of the proof is to show that in each case $\pi_{\kappa}$ is the desired proximity. 
(1) Suppose $\pi$ is an Efremovich proximity. Then there is a $T_{2}$-compactification $\kappa$ such that $\pi_{\kappa}=\pi$. (See Smirnov [4].) Thus $\kappa_{W}(\pi)$ is equivalent to a $T_{2}$-compactification of $X$.

(2) Let $\kappa$ be the usual Wallman compactification of $X$. We claim $\pi_{\kappa}=\pi_{W}$. This follows from the fact that if $\mathscr{Q} 6$ is an ultraclosed filter on $X$ and $A \subset X$ we have

$$
\mathscr{U} \in e_{W}(A)^{-} \text {iff } A^{-} \in \mathscr{C} \text {. }
$$

(3) Let $\kappa$ be the one-point compactification of $X$. We claim $\pi_{k}=\pi_{A}$. Let $\omega$ be the point at infinity. The key relation is that for $A \subset X$ we have $\omega \in e(A)^{-}$iff $A^{-}$is not compact.

3. Compact Wallman extensions. In this section we will look at a condition on $\pi$ which guarantees that the associated Wallman-type extension is compact. This condition states that certain large Wallman grills, called giant $\pi$-grills, must be $\pi$-clans. Covered proximities which satisfy this condition will be called compactification proximities. For these proximities the associated Wallman-type extensions are the weakly regular compactifications of Reed [2]. In fact, weakly regular covered principal $T_{1}$-compactifications turn out to be exactly those Wallman extensions whose induced proximities are compactification proximities.

We will spend some time studying the relation of weak regularity to what we have done here. We will obtain a characterization of weak regularity in terms of the associated nearness. We will also study the relation between weak regularity and Wallmancompleteness.

Finally, we will see that the operation of taking the Wallman extension is a bijection from the compactification proximities compatible with a given topology to the covered weakly regular principal $T_{1}$-compactifications of the space.

Definition 3.1. (i ) For subsets $A$ and $B$ of $X$ we say $A \delta_{\pi} B$ iff there is an ultraclosed filter $\mathscr{C}$ on $X$ such that $A \in \mathscr{C} \subset \pi(B)$.

(ii) If $\mathscr{A}$ is a family of subsets of $X$ then $\delta_{\pi}(\mathscr{A})=\left\{S: A \delta_{\pi} S\right.$ for all $A$ in $\mathscr{C}$ \}.

Next we will develop some properties of $\delta_{\pi}$. The following proposition shows that $\delta_{\pi}$ has many of the properties of $\pi$, but it is coarser than $\pi$.

Proposition 3.2. Let $A, B$, and $C$ be subsets of $X$

(i) $\varnothing \Phi_{\pi} A$ and $A \phi_{\pi} \varnothing$.

(ii) If $A \cap B \neq \varnothing$ then $A \delta_{\pi} B$. 
(iii) If $A \delta_{\pi} B$ and $B \subset C$ then $A \delta_{\pi} C$.

(iv) If $B \delta_{\pi} A$ and $B \subset C$ then $C \delta_{\pi} A$.

(v) If $A \delta_{\pi}(B \cup C)$ then $A \delta_{\pi} B$ or $A \delta_{\pi} C$.

(vi) $\delta_{\pi} \subset \pi$.

Corollary 3.3. If $\mathscr{F}$ is a filter on $X$ then $\delta_{\pi}(\mathscr{F})$ is a grill which contains $\mathscr{F}$.

Construction of $\nu_{c}(\pi)$. For any filter $\mathscr{F}$ let $\mathscr{F}^{i}$ be the filter generated by the open sets of $\mathscr{F}$. If $\mathscr{C}$ is an ultraclosed filter on $X$ we will call $\delta_{\pi}\left(\mathscr{C}^{i}\right)$ a giant $\pi$-grill. Then $\nu_{c}(\pi)$ is generated by all the giant $\pi$-grills on $X$; i.e., $\nu_{c}(\pi)$ consists of the families of the subsets of $X$ which are contained in some $\delta_{\pi}\left(\mathscr{U}^{i}\right)$.

Proposition 3.5.

(i) $\nu_{c}(\pi)$ is a nearness on $X$.

(ii) $\nu_{W}(\pi) \subset \nu_{c}(\pi)$.

(iii) If $\sigma$ is a $\pi$-clan which contains an ultraclosed filter $\mathscr{U}$ then $\sigma \subset \delta_{\pi}\left(\mathscr{U}^{i}\right)$.

Proof. To see that $\nu_{c}(\pi)$ is a nearness we note that for $x \in X$ we have $\{x\} \in \delta_{\pi}\left(\dot{x}^{i}\right)$. Thus each singleton is a member of one of the generating grills. This is sufficient to guarantee that $\nu_{c}(\pi)$ is a nearness. (See Gagrat and Thron [1], Thm. 2.7.)

It is clear that (ii) follows from (iii). To see that (iii) holds, let $S \in \sigma$, and let $G$ be an open set in $\mathscr{U}$. We need to show $G \delta_{\pi} S$. Since $G \in \mathscr{U}$, it is sufficient to show $\mathscr{U} \subset \pi(S)$. This last relation follows easily from the fact that $\mathscr{U} \subset \sigma$ and $\sigma$ is a $\pi$-clan.

THEOREM 3.6. The nearness $\nu_{c}(\pi)$ is contigual.

Proof. Let $\mathscr{A}$ be a family of subsets of $X$ such that every finite subfamily of $\mathscr{A}$ is a member of $\nu_{0}(\pi)$. Set

$$
\mathscr{K}=\left\{K: K \text { is closed and } \mathscr{A} \subset \delta_{\pi}(\sim K)\right\} \text {. }
$$

(i) $\mathscr{K}$ has the finite intersection property. Let $K_{1}, \cdots, K_{n} \in$ $\mathscr{K}$. For each $i$, choose $A_{i} \in \mathscr{A}$ so that $\sim K_{i} \phi_{\pi} A_{i}$. Now $\left\{A_{1}, \cdots, A_{n}\right\} \in$ $\nu_{c}(\pi)$, and so for some ultraclosed filter $\mathscr{C}$ we have

$$
\left\{A_{1}, \cdots, A_{n}\right\} \subset \delta_{\pi}\left(\mathscr{U}^{i}\right) .
$$

We claim that each $K_{i}$ is in $\mathscr{U}$, so that the $K_{i}$ 's intersect. Suppose some $K_{j}$ is not in $\mathscr{U}$. Then since $\mathscr{U}$ is ultraclosed we have $\sim K_{j} \in \mathscr{U}^{i}$. But $A_{j} \in \delta_{\pi}\left(\mathscr{U}^{i}\right)$, and so $\sim K_{j} \delta_{\pi} A_{j}$. But this violates 
the choice of $A_{j}$.

(ii) Let $\mathscr{V}$ be an ultraclosed filter containing $\mathscr{K}$. This exists, by Zorn's lemma. We claim $\mathscr{A} \subset \delta_{\pi}\left(\mathscr{V}^{i}\right)$.

Let $A \in \mathscr{A}$ and let $V$ be an open set in $\mathscr{V}$. Since $\mathscr{K} \subset \mathscr{V}$ we have $\sim V \notin \mathscr{K}$. But $\sim V$ is closed, and so we must have $\mathscr{A} \subset \delta_{\pi}(V)$. In particular then, $V \delta_{\pi} A$.

REMARK 3.7. We have constructed a contigual nearness using a Lodato proximity on $X$. It is not clear that this nearness is even compatible with the topology on $X$. Its closure operator may be too coarse; i.e., there may not be enough closed sets produced by the nearness. However, if $\nu_{c}(\pi)$ happens to land in the proximity class of $\pi$ then it turns out to be the Wallman nearness of $\pi$. In this case the Wallman-type extension is a compactification, and we will call $\pi$ a compactification proximity.

DeFinition 3.8. We say $\pi$ is a compactification proximity iff $\pi$ is covered and $\nu_{c}(\pi)$ is in the proximity class of $\pi$.

THEOREM 3.9. Let $\pi$ be a covered proximity. Then the following conditions are equivalent.

(i) $\pi$ is a compactification proximity.

(ii) $\delta_{\pi}\left(\mathscr{C}^{i}\right)$ is a $\pi$-clan for every ultraclosed filter $\mathscr{C}$ on $X$.

(iii) $\nu_{c}(\pi)=\nu_{W}(\pi)$.

Proof. Clearly (i) $\Rightarrow$ (ii). Now assume (ii) holds. Recall that $\nu_{W}(\pi) \subset \nu_{c}(\pi)$. Thus all we need to show is that each $\delta_{\pi}\left(\mathscr{C}^{i}\right)$ is a Wallman $\pi$-clan. This follows easily from (ii) and from the fact that $\mathscr{U} \subset \delta_{\pi}\left(\mathscr{U}^{i}\right)$.

Finally, suppose (iii) holds. Since $\pi$ is covered, $\nu_{W}(\pi)$ is in the proximity class of $\pi$. (See Thm. 1.9.) Thus (i) holds.

THEOREM 3.10. If $\pi$ is a compactification proximity then $\kappa_{W}(\pi)$ is compact.

Proof. Since contigual nearnesses give rise to compact extensions, it is sufficient to show that $\nu_{w}(\pi)$ is contigual. (See Reed [3], Thm. 1.24.) However, we have seen that $\nu_{c}(\pi)$ is contigual (Thm. 3.6). If $\pi$ is a compactification proximity then $\nu_{c}(\pi)=\nu_{W}(\pi)$, by Theorem 3.9 .

REMARK 3.11. The next few results lead up to a formulation of weak regularity in terms of the associated nearness. 
LEMMA 3.12. Let $\kappa=(e, Y)$ be an extension of $X$ and denote $\pi_{\kappa}$ by $\pi$. Let $\mathscr{U}$ be an ultraclosed filter on $X$ and $y \in Y$.

(i) $e(\mathscr{U}) \rightarrow y$ iff $\mathscr{U} \subset \tau(y)$.

(ii) If $\mathscr{U} \subset \tau(y)$ then $\tau(y) \subset \delta_{\pi}\left(\mathscr{U}^{i}\right)$.

LEMMA 3.13. Let $\kappa$ be a principal $T_{1}$-extension of $X$. If $\nu_{c}\left(\pi_{\kappa}\right) \subset$ $\nu_{\kappa}$ then $\kappa$ is wealely regular.

Proof. Let $\kappa=(e, Y)$ and let $\pi=\pi_{\kappa}$. Assume $\nu_{c}\left(\pi_{\kappa}\right) \subset \nu_{\kappa}$.

(1) If $\mathscr{U}$ is an ultraclosed filter on $X$ and $e(\mathscr{U}) \rightarrow y$ then $\tau(y)=\delta_{\pi}\left(\mathscr{U}^{i}\right)$.

Since $e(\mathscr{U}) \rightarrow y$ then $\tau(y) \subset \delta_{\pi}\left(\mathscr{C}^{i}\right)$ by Lemma 3.12. We need to show that the hypothesis on $\kappa$ guarantees $\delta_{\pi}\left(\mathscr{C}^{i}\right) \subset \tau(y)$. Since $\nu_{c}(\pi) \subset \nu_{\kappa}$ we have that $\delta_{\pi}\left(\mathscr{U}^{i}\right) \in \nu_{\kappa}$, and so $\delta_{\pi}\left(\mathscr{C}^{i}\right) \subset \tau(z)$ for some $z \in Y$. Therefore $\tau(y) \subset \tau(z)$. Since $\kappa$ is a principal extension, this guarantees $\mathscr{N}_{z} \subset \mathscr{N}_{y}$. Since $\kappa$ is a $T_{1}$-extension $y=z$.

(2) $\kappa$ is weakly regular.

Let $\mathscr{C}$ be an ultraclosed filter on $X$ such that $e(\mathscr{U}) \rightarrow y$. From (1) we have that $\tau(y)=\delta_{\pi}\left(\mathscr{C}^{i}\right)$. We need to show $e\left(\mathscr{Q}^{i}\right)^{*} \rightarrow y$.

Let $G$ be an open neighborhood of $y$. Since $\kappa$ is a principal extension, there is a subset $A$ of $X$ such that $y \in \sim e(A)^{-} \subset G$. Thus $A \notin \tau(y)=\delta_{\pi}\left(\mathscr{U}^{i}\right)$. Let $U$ be an open set in $\mathscr{Z}$ such that $U_{\phi_{\pi}} A$. We claim $e(U)^{*} \subset G$.

Let $z \in e(U)^{*}$. Then there is an ultraclosed filter $\mathscr{Y}$ such that $e(U) \in e(\mathscr{V}) \rightarrow z$. Then $U \in \mathscr{Y}$, and so $\mathscr{V} \not \subset \pi(A)$, since $U \delta_{\pi} A$. Pick $V \in \mathscr{Y}$ so that $A \pi V$. Then by definition $e(A)^{-} \cap e(V)^{-}=\varnothing$. Since $e(\mathscr{Y}) \rightarrow z$ we have $z \in e(V)^{-}$. Thus $z \in \sim e(A)^{-} \subset G$. We have shown $e(U)^{*} \subset G$.

LEMMA 3.14. If $\kappa$ is a weakly regular compactification then $\nu_{\kappa} \supset \nu_{c}\left(\pi_{\kappa}\right)$.

Proof. Let $\kappa=(e, Y)$ and $\pi=\pi_{\kappa}$. We need to show $\delta_{\pi}\left(\mathscr{Q}^{i}\right) \in \nu_{\kappa}$ for every ultraclosed filter $\mathscr{C}$. Let $\mathscr{C}$ be given. Since $Y$ is compact, we have $e(\mathscr{C}) \rightarrow y$ for some $y \in Y$. We claim $\delta_{\pi}\left(\mathscr{C}^{i}\right) \subset \tau(y)$.

Let $A \in \delta_{\pi}\left(\mathscr{C}^{i}\right)$. We wish to show $y \in e(A)^{-}$. Let $G$ be an open neighborhood of $y$. We will show $G \cap e(A) \neq \varnothing$.

Since $e(\mathscr{C}) \rightarrow y$ we have by weak regularity that $e\left(\mathscr{C}^{i}\right)^{*} \rightarrow y$. Hence we can choose an open member $U$ in $\mathscr{C}$ such that $e(U)^{*} \subset G$. Since $A \in \delta_{\pi}\left(\mathscr{U}^{i}\right)$ we have $U \delta_{\pi} A$. Let $\mathscr{Y}$ be an ultraclosed filter such that $U \in \mathscr{V} \subset \pi(A)$.

Since $\kappa$ is a compactification of $X, \nu_{\kappa}$ is contigual. (See Reed 
[3], Thm. 1.25.) Thus from $\mathscr{V} \subset \pi(A)$ we can conclude $\mathscr{V} \cup\{A\} \in \nu_{\kappa}$. Choose $z \in Y$ so that $\mathscr{V} \cup\{A\} \subset \tau(z)$. Clearly then $e(\mathscr{V}) \rightarrow z$. Thus $z \in e(U)^{*} \subset G$. But we also have $A \in \tau(z)$, so that $z \in e(A)^{-}$. Thus $G \cap e(A) \neq \varnothing$.

THEOREM 3.15. Let $\kappa$ be a principal $T_{1}$-compactification of $X$. Then $\kappa$ is weakly regular iff $\nu_{c}\left(\pi_{\kappa}\right) \subset \nu_{k}$.

Proof. This follows immediately from Lemmas 3.14 and 3.13.

Next we will look at the relation between weak regularity and the associated proximity.

THEOREM 3.16. If $\pi$ is a compactification proximity, then $\kappa_{W}(\pi)$ is a covered weakly regular principal $T_{1}$-compactification of $X$.

Proof. The theorem simply gathers up some previous results. We have already seen that $\kappa_{W}(\pi)$ is a covered principal $T_{1}$-extension (Cor. 2.12). If $\pi$ is a compactification proximity, then $\kappa_{W}(\pi)$ is a compactification (Thm. 3.10). Moreover, $\nu_{c}(\pi)=\nu_{W}(\pi)$ by Theorem 3.9. Thus by Lemma 3.13 we have that $\kappa_{W}(\pi)$ is weakly regular.

This now allows us to obtain a new characterization of Wallman nearnesses whose induced proximities are compactification proximities.

THEOREM 3.17. If $\pi$ is a compactification proximity then $\nu_{W}(\pi)$ is the smallest nearness in the proximity class of $\pi$ which is induced by a weakly regular compactification of $X$.

Proof. Let $\kappa$ be a weakly regular compactification of $X$ such that $\nu_{\kappa}$ is in the proximity class of $\pi$. Note then $\pi_{\kappa}=\pi$. We wish to show $\nu_{W}(\pi) \subset \nu_{\kappa}$. Recall $\nu_{W}(\pi) \subset \nu_{c}(\pi)$ by Proposition 3.5. Also since $\kappa$ is weakly regular we have $\nu_{c}(\pi) \subset \nu_{\kappa}$ (Lemma 3.14). Since $\pi=\pi_{\kappa}$ we have the desired result.

We note that $\nu_{W}(\pi)$ is induced by a weakly regular compactification; namely, $\kappa_{W}(\pi)$ (Thm. 3.16).

Lemma 3.18. If $\kappa$ is a covered extension then $\nu_{\kappa} \subset \nu_{W}\left(\pi_{\kappa}\right)$.

Proof. Since $\kappa$ is covered we have that each $\tau(y)$ is a Wallman $\pi_{k}$-clan (Lemma 2.12). Thus $\nu_{k} \subset \nu_{W}\left(\pi_{k}\right)$.

Corollary 3.19. Compactification proximities on $X$ are those proximities which are induced by covered weakly regular compactifications of $X$. 
Proof. Let $\pi$ be a compactification proximity. Then $\kappa_{W}(\pi)$ is a covered weakly regular compactification of $X$, by Theorem 3.16. But $\pi$ is induced by $\kappa_{W}(\pi)$, by Theorem 2.11 .

Conversely, let $\kappa$ be a covered weakly regular compactification of $X$. We wish to show $\pi_{\kappa}$ is a compactification proximity. Note $\pi_{\kappa}$ is covered, by Lemma 2.12. We will show $\nu_{c}\left(\pi_{\kappa}\right)=\nu_{W}\left(\pi_{\kappa}\right)$.

Recall $\nu_{W}\left(\pi_{\kappa}\right) \subset \nu_{c}\left(\pi_{\kappa}\right)$. Since $\kappa$ is a weakly regular compactification, we have $\nu_{c}\left(\pi_{\kappa}\right) \subset \nu_{\kappa}$ (Lemma 3.14). Since $\kappa$ is covered, $\nu_{\kappa} \subset$ $\nu_{W}\left(\pi_{\kappa}\right)$ by the preceding lemma. This establishes the desired result. By Theorem 3.9 then $\pi_{\kappa}$ is a compactification proximity.

Next we will investigate the relation between weakly regular compactifications and Wallman-type extensions. In order to simplify the discussion we will introduce the notion of an efficient extension.

DEFinition 3.20. An efficient extension of $X$ is a covered principal $T_{1}$-extension.

LEMMA 3.21. Every weakly regular principal compactification of $X$ is Wallman-complete.

Proof. Let $\kappa$ be a weakly regular principal compactification of $X$. We wish to show that $\kappa$ is Wallman-complete. Since $\kappa$ is a principal extension of $X$, it is sufficient to show $\nu_{W}\left(\pi_{\kappa}\right) \subset \nu_{\kappa}$, by Theorem 2.5. Now $\nu_{W}\left(\pi_{\kappa}\right) \subset \nu_{c}\left(\pi_{\kappa}\right)$ by Proposition 3.5. But since $\kappa$ is a weakly regular compactification we have $\nu_{c}\left(\pi_{\kappa}\right) \subset \nu_{\kappa}$ by Lemma 3.14 .

This says that for principal compactifications of $X$, weak regularity implies Wallman-completeness. Next we will show that for a Wallman-complete (principal $T_{1}$ ) compactification to be weakly regular the associated proximity must be a compactification proximity.

THEOREM 3.22. Let $\kappa$ be an efficient compactification of $X$. Then $\kappa$ is weakly regular iff $\kappa$ is Wallman-complete and $\pi_{\kappa}$ is a compactification proximity.

Proof. $\Leftrightarrow$ Suppose $\kappa$ is weakly regular. Then $\kappa$ is Wallmancomplete, by the preceding result. Since $\kappa$ is a covered weakly regular compactification, we have that $\pi_{\kappa}$ is a compactification proximity, by Corollary 3.19.

$\Leftrightarrow$ Suppose $\kappa$ is Wallman-complete and $\pi_{\kappa}$ is a compactification proximity. To show $\kappa$ is weakly regular it is sufficient to show $\nu_{c}\left(\pi_{\kappa}\right) \subset \nu_{\kappa}$ (Lemma 3.13). Since $\pi_{\kappa}$ is a compactification proximity we have $\nu_{c}\left(\pi_{\kappa}\right)=\nu_{W}\left(\pi_{\kappa}\right)$. But $\kappa$ is Wallman-complete, and so $\nu_{W}\left(\pi_{\kappa}\right) \subset$ $\nu_{\kappa}$, (Thm. 2.5). 
COROLLARY 3.23. Every efficient weakly regular compactification $\kappa$ of $X$ is a Wallman-type extension.

Proof. From the preceding corollary we have that $\kappa$ is Wallmancomplete. Since $\kappa$ is also covered, it must be a Wallman-type extension (Thm. 2.9).

Thus in the context of efficient compactifications, weakly regular extensions are Wallman-type extensions.

To complete this section we will show that $\kappa_{W}$ defines a correspondence between compactification proximities and weakly regular efficient compactifications.

THEOREM 3.24. $\kappa_{W}$ is a bijection from the compactification proximities on $X$ to the efficient weakly regular compactifications of $X$.

Proof. From Theorem 3.16 we have that $\kappa_{W}$ maps compactification proximities into efficient weakly regular compactifications. We know from Theorem 2.11 that $\kappa_{W}$ is $1-1$. Finally, let $\kappa$ be an efficient weakly regular compactification of $X$. From Corollary 3.23 we have $\kappa$ is a Wallman-type extension. Thus by Theorem 2.11 we have $\kappa$ equivalent to $\kappa_{W}\left(\pi_{\kappa}\right)$. But $\pi_{\kappa}$ is a compactification proximity, by Theorem 3.22 .

Open question 3.25. We have established some conditions under which a Wallman-type extension is compact; namely, it is compact if all grills $\delta_{\pi}\left(\mathscr{U}^{i}\right)$, are $\pi$-clans, where $\mathscr{U}$ is an ultraclosed filter. It would be of interest to obtain other conditions on $\pi$ which would guarantee that $\kappa_{W}(\pi)$ is compact.

\section{REFERENCES}

1. M.S. Gagrat and W.J. Thron, Nearness structures and proximity extensions, Trans. Amer. Math. Soc., 208 (1975), 103-125.

2. Ellen E. Reed, $A$ class of $T_{1}$-compactifications, Pacific J. Math., 65 (1976), 471-484. 3. - Nearnesses, proximities, and $T_{1}$-compactifications, Trans. Amer. Math. Soc., 236 (1978), 193-207.

4. Y. M. Smirnov, On proximity spaces, Mat. Sb., 21 (1952), 543-574, English translation A. M. S. Transl. Series 2, 38 (1964), 5-35.

5. W. J. Thron, Topological Structures, Holt, Rinehart and Winston, New York, 1966.

Received December 21, 1979 and in revised from March 14, 1980.

ST. MoRy's COLLEge

Notre DAME, IN 46556 



\section{PACIFIC JOURNAL OF MATHEMATICS}

\section{EDITORS}

DONALD BABBITT (Managing Editor)

University of California

Los Angeles, CA 90024

HUGo RossI

University of Utah

Salt Lake City, UT 84112

C. C. MOORE and ANDREW OGG

University of California

Berkeley, CA 94720
J. DugundjI

Department of Mathematics

University of Southern California

Los Angeles, CA 90007

R. FinN and J. Milgram

Stanford University

Stanford, CA 94305

\section{ASSOCIATE EDITORS}
R. ARENS
E. F. BECKENBACH
B. H. NeumanN
F. WOLF
K. YOSHIDA

\section{SUPPORTING INSTITUTIONS}

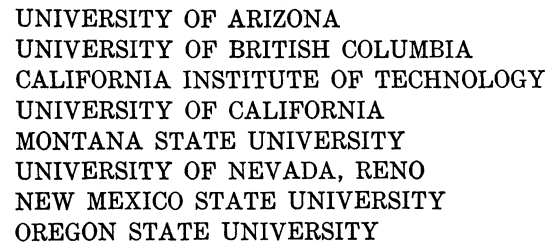

UNIVERSITY OF ARIZONA

UNIVERSITY OF BRITISH COLUMBIA

CALIFORNIA INSTITUTE OF TECHNOLOGY

UNIVERSITY OF CALIFORNIA

MONTANA STATE UNIVERSITY

UNIVERSITY OF NEVADA, RENO

NEW MEXICO STATE UNIVERSITY

OREGON STATE UNIVERSITY

\author{
UNIVERSITY OF OREGON \\ UNIVERSITY OF SOUTHERN CALIFORNIA \\ STANFORD UNIVERSITY \\ UNIVERSITY OF HAWAII \\ UNIVERSITY OF TOKYO \\ UNIVERSITY OF UTAH \\ WASHINGTON STATE UNIVERSITY \\ UNIVERSITY OF WASHINGTON
}

The Supporting Institutions listed above contribute to the cost of publication of this Journal, but they are not owners or publishers and have no responsibility for its content or policies.

Mathematical papers intended for publication in the Pacific Journal of Mathematics should be in typed form or offset-reproduced, (not dittoed), double spaced with large margins. Please do not use built up fractions in the text of the manuscript. However, you may use them in the displayed equations. Underline Greek letters in red, German in green, and script in blue. The first paragraph or two must be capable of being used separately as a synopsis of the entire paper. Please propose a heading for the odd numbered pages of less than 35 characters. Manuscripts, in triplicate, may be sent to any one of the editors. Please classify according to the scheme of Math. Reviews, Index to Vol. 39. Supply name and address of author to whom proofs should be sent. All other communications should be addressed to the managing editor, or Elaine Barth, University of California, Los Angeles, California, 90024.

50 reprints to each author are provided free for each article, only if page charges have been substantially paid. Additional copies may be obtained at cost in multiples of 50 .

The Pacific Journal of Mathematics is issued monthly as of January 1966. Regular subscription rate: $\$ 102.00$ a year (6 Vols., 12 issues). Special rate: $\$ 51.00$ a year to individual members of supporting institutions.

Subscriptions, orders for numbers issued in the last three calendar years, and changes of address shoud be sent to Pacific Journal of Mathematics, P.O. Box 969, Carmel Valley, CA 93924, U.S.A. Old back numbers obtainable from Kraus Per!odicals Co., Route 100, Millwood, NY 10546.

PUBLISHED BY PACIFIC JOURNAL OF MATHEMATICS, A NON-PROFIT CORPORATION

Printed at Kokusai Bunken Insatsusha (International Academic Printing Co., Ltd.). 8-8, 3-chome, Takadanobaba, Shinjuku-ku, Tokyo 160, Japan. 


\section{Pacific Journal of Mathematics}

\section{Vol. 95, No. $2 \quad$ October, 1981}

George E. Andrews, The Rogers-Ramanujan reciprocal and Minc's

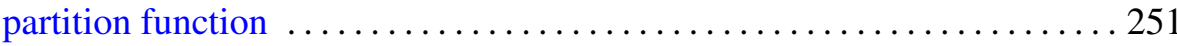

Allan Calder, William H. Julian, Ray Mines, III and Fred Richman,

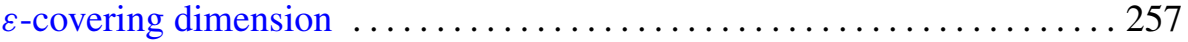

Thomas Curtis Craven and George Leslie Csordas, An inequality for the distribution of zeros of polynomials and entire functions $\ldots \ldots \ldots \ldots 263$

Thomas Jones Enright and R. Parthasarathy, The transfer of invariant

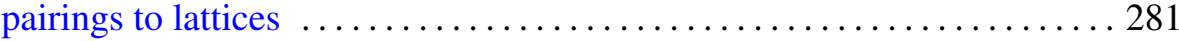

Allen Roy Freedman and John Joseph Sember, Densities and

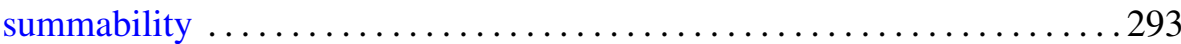

Robert Heller and Francis Aubra Roach, A generalization of a classical necessary condition for convergence of continued fractions . . . . . . 307

Peter Wilcox Jones, Ratios of interpolating Blaschke products ........... 311

V. J. Joseph, Smooth actions of the circle group on exotic spheres ........ 323

Mohd Saeed Khan, Common fixed point theorems for multivalued

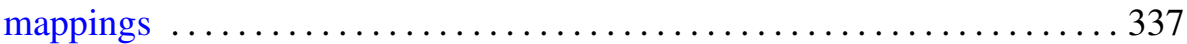

Samuel James Lomonaco, Jr., The homotopy groups of knots. I. How to

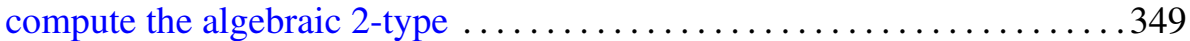

Louis Magnin, Some remarks about $C^{\infty}$ vectors in representations of connected locally compact groups ............................ 391

Mark Mandelker, Located sets on the line . . . . . . . . . . . . . . . . . 401

Murray Angus Marshall and Joseph Lewis Yucas, Linked quaternionic mappings and their associated Witt rings $\ldots \ldots \ldots \ldots \ldots \ldots \ldots \ldots . \ldots \ldots 11$

William Lindall Paschke, $K$-theory for commutants in the Calkin algebra

W. J. Phillips, On the relation $P Q-Q P=-i I$ 435

Ellen Elizabeth Reed, A class of Wallman-type extension. 443

Sungwoo Suh, The space of real parts of algebras of Fourier transforms 461 Antonius Johannes Van Haagen, Finite signed measures on function

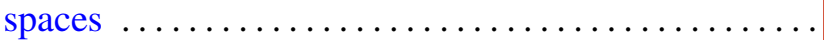

Richard Hawks Warren, Identification spaces and unique uniformity 